\title{
Efectividad de la férula U-Mano en el tratamiento del síndrome del túnel del carpo
}

\author{
Claudia Andrea Urbina*
}

\section{Resumen}

El síndrome del túnel del carpo (STC) es el trauma acumulativo más frecuente del mundo y la patología que los usuarios de computador sufren con mayor regularidad; es así como en terapia ocupacional este síndrome constituye un gran porcentaje de los motivos de consulta.

El tratamiento terapéutico del STC, tiene como objetivo minimizar la inflamación, reducir el dolor y mantener el deslizamiento normal de los tendones flexores dentro del túnel del carpo, mediante un programa, modificación de la actividad, técnicas de protección articular y de conservación de energía.

El objetivo del presente trabajo es evaluar la efectividad de la férula U-Mano para el tratamiento del STC, basandose en la relación costo/beneficio para los pacientes, en comparación con la férula convencional utilizada en el medio.

Palabras clave: Sindrome del túnel del carpo, ergonómica, intervención ocupacional, protección articular, férula, Manú.

\section{Effectivity of the U. Splint-Hand in the treatment of the carpal tunnel syndrome}

\section{Abstract}

The Carpal Tunnel Syndrome (STC) (CTS) is the most frequent accumulative trauma in the world and the most common pathology on computer users; in Occupational Therapy this syndrome has a high percentage of consultation.

The objective of the therapeutic treatment of the CTS is to minimize inflammation effects, reduce pain and maintain the normal movement of flexor tendons within the carpal tunnel, with a splint program, modification of activity, joint protection techniques and maintenance of energy.

Terapista ocupacional, Especialista en Rehabilitación de Mano y Miembro Superior. Decana, Facultad de Terapia Ocupacional Institución Universitaria Escuela Colombiana de Rehabilitación. tocupacional@ecr.edu.co 
The fundamental purpose of the herein work is to verify the effectiveness of the Hand U splint for the treatment of the STC, determining if it offers a favorable cost/benefit relation for patients compared with the conventional splint.

Key words: carpal tunnel syndrome, ergonomics, occupational intervention, joint protection, STC splint, hand.

\section{Introducción}

El STC es una patología que afecta la mano, provocada por el aumento de presión en el túnel del carpo produciéndose compresión del nervio mediano en su paso a través del túnel del carpo ${ }^{1}$.

La aparición de este síndrome puede darse luego de un trauma en la muñeca, o iniciarse lentamente en personas con exposición manual a trauma y movimientos repetitivos. Las lesiones por esfuerzos repetitivos, como es el STC se han incrementado por el empleo creciente de los computadores, convirtiéndola en la enfermedad profesional más frecuente a nivel mundial.

En Estados Unidos, su incidencia es de 1 a 3 sujetos por cada 1.000 habitantes cada año. En grupos de riesgo, esta incidencia se incrementa a 150 casos por 1.000 habitantes y su prevalencia puede llegar a 500 casos por 1.000 sujetos anualmente. $\mathrm{Ci}$ fras similares se observan en países industrializados como Holanda y en Gran Bretaña. En cuanto al sexo, es más frecuente en mujeres 3 a 1 y en los grupos de edad entre 45 a 60 años, con una baja frecuencia en menores de 30 años.

En Colombia, el síndrome del túnel del carpo (STC) es el diagnóstico más frecuentemente reportado por las empresas promotoras de salud, con un promedio de $27 \%$ del total de los casos reportados por enfermedad profesional ${ }^{2}$.

Cailliet, R, Síndromes dolorosos de la mano. Editorial El Manual Moderno. 1985

Rancel, Carlos Eduardo. STC: diagnóstico y tratamiento. Revista ARP Colpatria Año 8 / Edición 32 / Diciembre de 2005.
El síndrome del túnel del carpo tiene consecuencias que van más allá de lo laboral. Puede generarse en el trabajo pero sus consecuencias se sienten en la vida cotidiana ya que los pacientes ven afectada la posibilidad de "agarrar" objetos y de tomar elementos con fuerza o sostener cosas livianas; todo ello es molesto e incapacita para las actividades diarias. El síndrome del conducto carpiano o túnel del carpo produce: dolor e incapacidad, pérdida de fuerza en las manos, deterioro en el desarrollo de actividades cotidianas que requieran de la utilización de las manos ${ }^{3}$.

Entre los síntomas más frecuentes que se observan en los pacientes son: Hormigueo de los tres primeros dedos, parestesias nocturnas, disestesias o hiperestesias provocadas por el frío, irradiaciones a nivel proximal sobre la zona por donde transcurre el nervio mediano, anestesia de los dedos pulgar, índice, medio y mitad radial del anular, disminución de la sensibilidad de los dedos índice, medio y anular, pérdida de destreza motriz fina, en una etapa tardía hay atrofia de la región tenar y pérdida de la coordinación para oponer el pulgar. Se produce alivio del dolor al inmovilizar la muñeca en posición neutra.

Dentro de los tratamientos a utilizar en la rehabilitación del síndrome estarían: el tratamiento no quirúrgico o conservador, cirugía, terapia física, y/o ocupacional, observando la etapa en que se encuentre el usuario para su rehabilitación ${ }^{4}$. Si el

Szabo, M.D. Acute carpal tunnel syndrome. Hand Clinics. Vol. 14, № 3, agosto, 1998, págs. 419-427.

Mcnaugthon, M.D. Overuse syndrome/repetitive strain injury: the occupational therapit's role. British Journal of Occupational Therapy. Febrero, 1997. 
STC tiene relación con el trabajo, una primera aproximación sería intentar modificar las condiciones de éste para disminuir la irritación del nervio.

Existen férulas que se utilizan para disminuir los síntomas del STC: La férula convencional para el túnel del carpo, es una férula corta, volar, elaborada en material termoplástico moldeable a bajas temperaturas, que mantiene la articulación de la muñeca en 10 a $20^{\circ}$ de extensión, pues según estudios se ha comprobado que en esta posición y con uso permanente los pacientes tienen disminución en la sintomatología, permitiendo la oposición del pulgar y la movilización libre de todos los demás dedos $^{5}$.(Figura 1) La férula tradicional, palmeta corta para el STC, estabiliza la muñeca, manteniéndola en la posición ya mencionada. Su uso reduce el dolor y el edema dentro del túnel ${ }^{6,7}$.

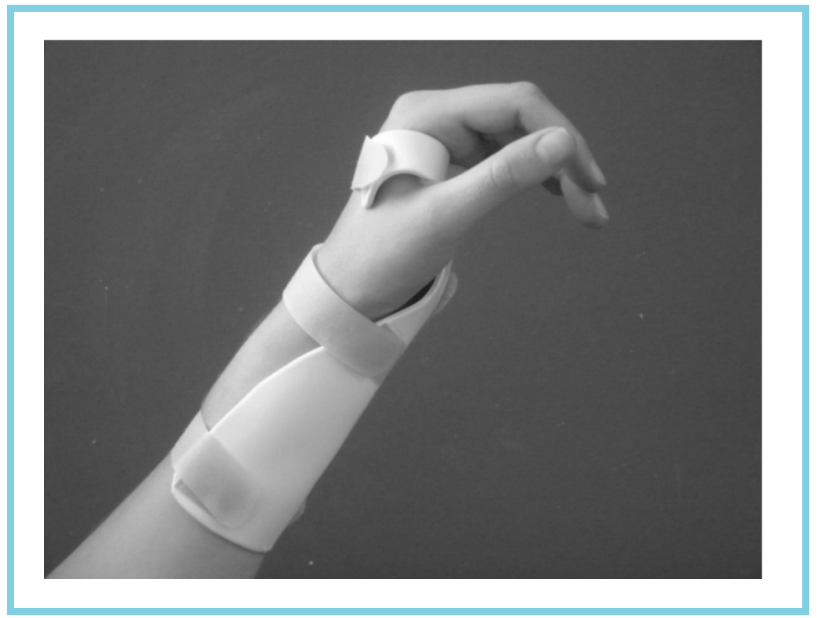

Figura 1. Férula convencional.

La férula "U-Mano" para pacientes con STC, puede describirse como una férula confeccionada en material suave basada en la Manu®, sin com-

Fees, Philip. Hand splinting principles and methods. 1990.

6 Santaella y Herrera. Comparación de dos férulas de distinto material en cuanto a efectividad en amas de casa con STC. Revista de la Ocupación Humana, Vol. 7, N 4. 1998, págs. 13-37.

Sailer, O.T. The role of splinting and rehabilitation in the treatment of carpal and cubital tunnel syndromes. Hand Clinics, Vol. 12, º 2, Mayo, 1996, págs. 223- 240. ponentes rígidos sujetada con correas de velcro alrededor de las cabezas de los cuatro últimos metacarpianos, constituida por un rectángulo del tamaño de los cuatro últimos dedos, en donde por una hendidura horizontal, del tamaño del ancho de los dedos tercero y cuarto, se puedan introducir los mismos, quedando por encima, y dejando los dedos segundo y quinto, por fuera y por debajo ${ }^{8}$. El objetivo de esta férula es disminuir el volumen que ocupan los músculos flexores de los dedos dentro del túnel del carpo, disminuyendo así la presión intracarpal.

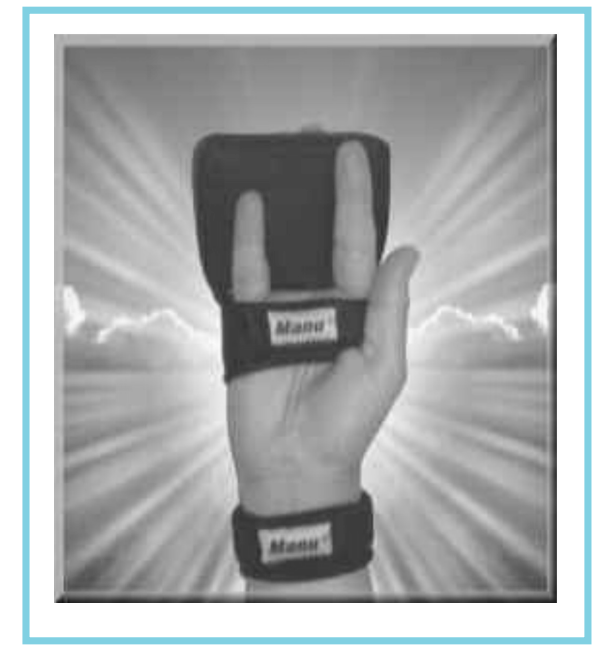

Figura 2. Férula Manú.

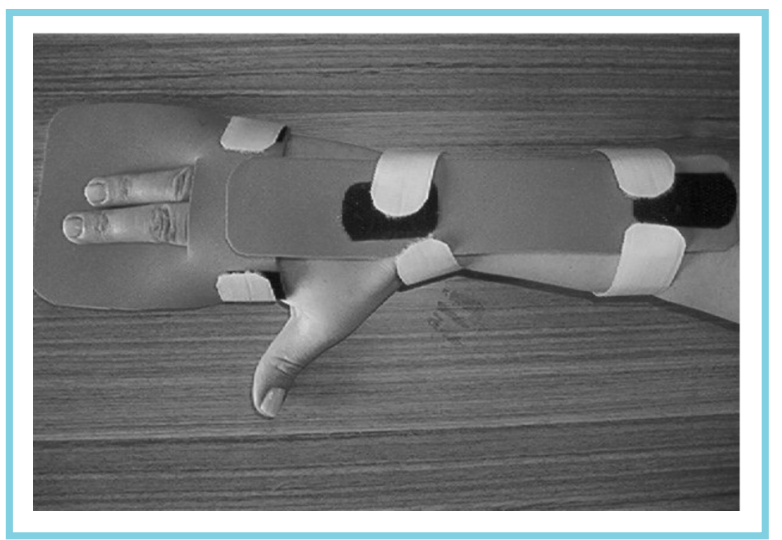

Figura 3. Férula U-Mano.

\footnotetext{
Manente, M.D. Ann innovative hand brace for carpal tunnel syndrome: a randomized controlled trial. Muscle and Nerve Journal. Agosto, 2001, págs. 1020-1025.
} 
El objetivo del estudio fue evaluar el costo/beneficio de la férula convencional vs. la férula UManú para el tratamiento de los pacientes con síndrome del túnel.

\section{Método}

El estudio fue dividido en dos fases; una primera en donde se determinó todo lo relacionado con el costo de cada una de las férulas, determinándose precios de los materiales, de la elaboración, la duración y la comodidad manifestada por los pacientes de su uso ${ }^{9}$.

La segunda fase correspondió a un estudio experimental de diseño preprueba - posprueba con dos mediciones en dos grupos ${ }^{10}$ con diferente intervención ocupacional; que diagramáticamente se representó así:
A G1
$01 \mathrm{X} 1$
$\mathrm{O} 2$
$\mathrm{X} 1 \mathrm{O} 3$
A G2
$01 \quad \mathrm{X} 2$
$\mathrm{O} 2$
$\mathrm{X} 2 \mathrm{O} 3$

das; como parte de la evaluación ocupacional se midió la independencia en la realización de las actividades de la vida diaria, mediante la aplicación del Test de Rodríguez y Arango.

El estudio incluyó 22 pacientes con síndrome del túnel del carpo bilateral diagnosticado por medio de electromiografía reciente, es decir, 44 muñecas con esta patología; se excluían los pacientes con STC a quienes se les hubiera practicado cualquier procedimiento quirúrgico en mano o antebrazo, que tuviera antecedentes traumáticos en el miembro superior comprometido. que presenten cualquier condición clínica causante de neuropatía que tuviese pacientes con STC secundario a alguna enfermedad de base como diabetes, polisacaridosis, amiloidosis y otras similares y que estuvieran en estado en embarazo.

20 pacientes fueron mujeres y 2 hombres. En el grupo 1 (férula convencional), se incluyeron 1 hombre y 10 mujeres $(9,1 \%$ - 90,9\%), al igual que en el grupo 2 (férula U-Mano, 9,1\% - 90,9\%). La distribución de profesiones correspondió en el grupo 1

$\begin{array}{ll}\text { Amas de casa } & 5(45,4 \%) \\ \text { Enfermeras } & 3(27,3 \%) \\ \text { Secretarias } & 2(18,2 \%) \\ \text { Pensionado sastrería } & 1(9,1 \%)\end{array}$

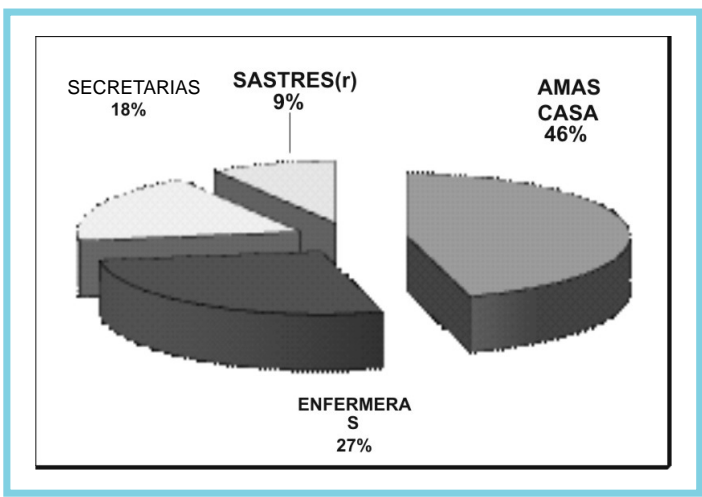

Gráfica 1. Ocupación de los usuarios con la férula convencional Cromwen, Florence. Hand rehabilitation in occupational
therapy. 2000.
Se realizaron tres valoraciones individuales del STC: una al inicio, otra a la tercera semana que se denominó intermedia y una última después de la sexta semana llamada final. Cada valoración incluía las pruebas de Tinel, Phalen y Flick, el test de monofilamentos, examen muscular manual, el trofismo muscular de la eminencia tenar, el dolor, la movilidad del pulgar y la funcionalidad de los patrones integrales de las manos afecta-

\footnotetext{
Hernández, Sampieri, Metodología de la investigación. McGraw-Hill. Tercera edición. México. 2003.

10 Polit, Investigación Científica en Ciencias de la Salud. McGrawHill. Sexta edición, México. 2000.
} 
En el grupo 2 las ocupaciones fueron:
Amas de casa
$3(27,3 \%)$
Enfermeras
$3(27,3 \%)$
Empleadas servicio doméstico
$3(27,3 \%)$
Delineante arquitectura
$1(9,1 \%)$
Ingeniero mecánico
$1(9,1 \%)$

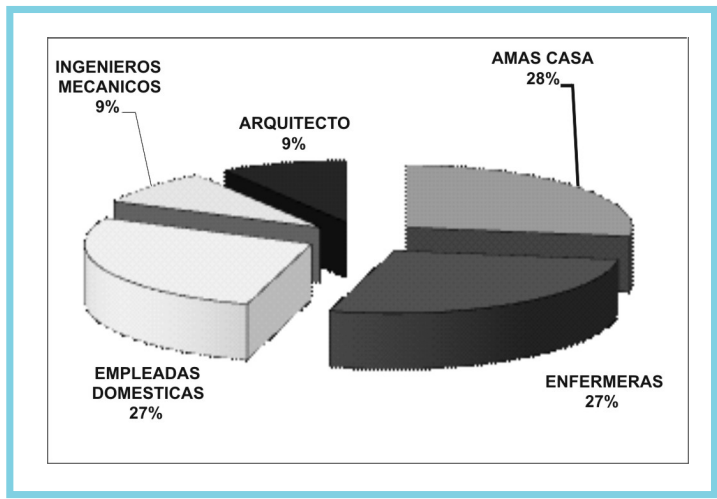

Gráfica 2. Ocupación de los usuarios férula U-Mano

\section{Procedimiento}

Seguido de la valoración inicial los grupos de pacientes fueron intervenidos ocupacionalmente con la utilización de las técnicas propias del enfoque biomecánico y cognoscitivo comportamental ${ }^{11}$, se diseñaron y aplicaron respectivamente la férula convencional y la U-Mano bilaterales. Se dieron las instrucciones de su intensidad de uso y cuidado y se controló de manera telefónica que estas instrucciones se cumplieran; a las 3 semanas se citaron los pacientes para llevar a cabo la valoración intermedia. Pasadas 6 semanas en cada uno de los casos, se citó a cada paciente para llevar a cabo la valoración final. Sólo 6 de los pacientes (12 muñecas) de los 11 iniciales del grupo 1 contestaron las llamadas telefónicas de control y asistieron a la valoración final. Los 11 usuarios que asistieron a la valoración inicial del grupo 2, contestaron los controles telefónicos y asistieron a la valoración final.

La intervención terapéutica incluyó la realización de ejercicios de fortalecimiento y estiramiento, así como la realización de actividades con propósito en donde se pudieran poner en práctica las técnicas de ahorro de energía y de protección articular.

\section{Resultados}

\section{Primera fase (costo)}

Para la primera fase se determinó el costo en pesos de la férula, el tiempo en minutos de la elaboración de la misma, la comodidad expresada por el usuario, el mantenimiento, duración, los equipos y herramientas que se usan para su construcción. Los resultados se muestran en la gráfica 3.

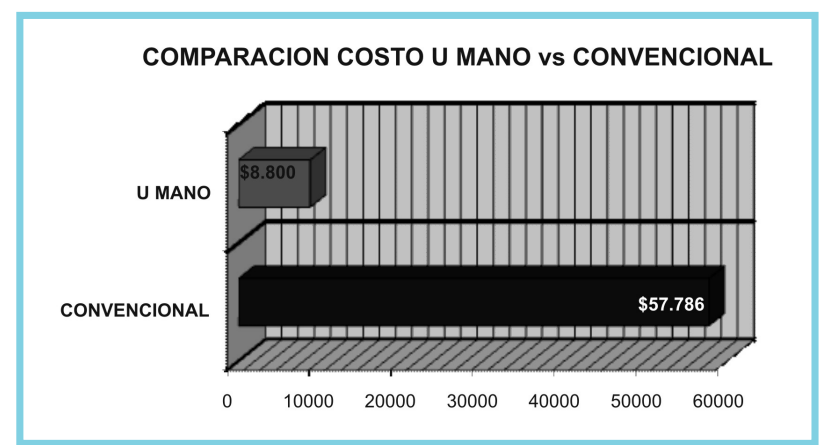

Gráfica 3. Comparación costo férula U-Mano vs. férula convencional en pesos

El precio total de cada una de las férulas fue calculado teniendo en cuenta el precio al momento de los materiales, el costo de una sesión de elaboración de material ortésico a precio de costo de sesión para empresa de prestación de servicios de salud estatal, el costo de la utilización de herramientas y equipos y el valor de los materiales secundarios de la construcción. A continuación una referencia de los valores encontrados (tabla 1).

\section{TABLA 1}

VALOR EN PESOS DE LAS FÉRULAS CONVENCIONAL Y U-MANO

\begin{tabular}{l|c|l|c|c|c}
\hline & Material & Otros & Sesiones & Equipos & Total \\
\hline Convencional & $\$ 24.375$ & $\$ 211$ & $\$ 32.000$ & $\$ 1.200$ & $\$ 57.786$ \\
U-Mano & $\$ 2.400$ & $\$ 180$ & $\$ 6.200$ & $\$ 20$ & $\$ 8.800$ \\
\hline
\end{tabular}

En la fase II se determinó el beneficio recibido por los pacientes con STC tras la utilización del respectivo material ortésico; se determinó entonces la atrofia de la eminencia tenar, la movilidad articular del pulgar, la funcionalidad de los agarres livianos, medianos y livianos, en términos de amplitud articular, fuerza, velocidad, destreza y propósito; 
el dolor mediante la Escala Verbal Análoga del dolor, la sensibilidad superficial, las disestesias y parestesias $^{12}$.

Las manos derechas de ambos grupos mostraban atrofia ligera de la eminencia tenar con relación a las manos izquierdas, y esta observación fue constante en las tres mediciones de cada uno de los pacientes de ambos grupos.

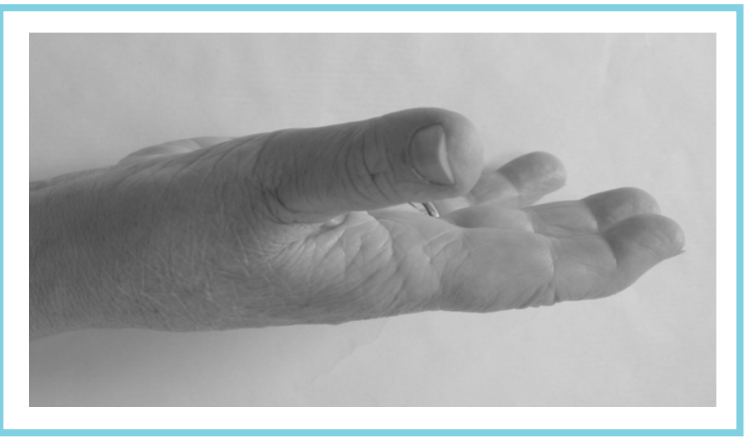

Figura 3. Atrofia eminencia tenar.

La movilidad de los pulgares se determinó llevando a cabo la prueba de movilidad del pulgar en grados del $1^{\circ}$ al $8^{\circ}$, correspondiente a tocar con el pulpejo del pulgar desde el pulpejo del dedo 2 progresivamente hacia cubital el pulpejo de los dedos 3, 4 y 5, la falange intermedia, la proximal en su cara volar del 5 dedo, y la cabeza del quinto metacarpiano en la cara palmar. Todos los pulgares se movieron 8/8 en ambos grupos.

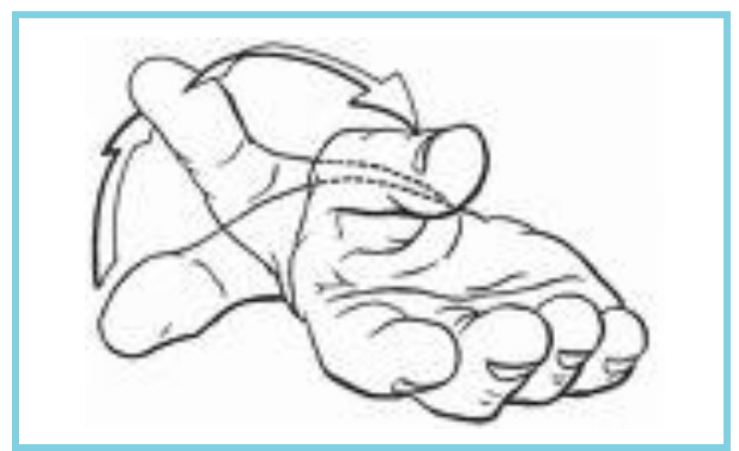

Gráfica 4. Prueba de movilidad del pulgar

Stanley, Barbara. 1992. Concepts in hand rehabilitation.
Se determinó si los patrones integrales eran funcionales, semifuncionales o no funcionales. Los agarres pesados y medianos (mano llena, gancho, cilíndrico) fueron funcionales en todos los casos. En los agarres livianos, correspondientes a las pinzas, se encontró diferencia tanto en el grupo de pacientes que usaron la férula convencional como en el grupo de pacientes que utilizaron la férula UMano. La funcionalidad se calculó teniendo en cuenta el propósito (10 puntos), la amplitud articular (10 puntos) la fuerza (10 puntos), la velocidad en la ejecución del patrón (10 puntos) y la destreza (10 puntos), para un total de 50 puntos (máxima calificación). Se plantearon tres rangos así:

\section{0 - $10 \quad$ No funcional \\ 11 - $30 \quad$ Semifuncional \\ 31 - $50 \quad$ Funcional}

La valoración intermedia arrojó iguales resultados a la valoración inicial de todos los pacientes, por lo tanto, no aparece registro de la misma. Aparecen los resultados de la primera valoración en la primera columna de cada mano, y en la segunda columna de cada mano aparece la información de la valoración final.

En ambos grupos de observó un aumento de la funcionalidad en las manos derechas: en el grupo de la férula U-Mano de 4 puntos y en la férula convencional de 2 . Las manos izquierdas estaban con $50 / 50$ de funcionalidad.

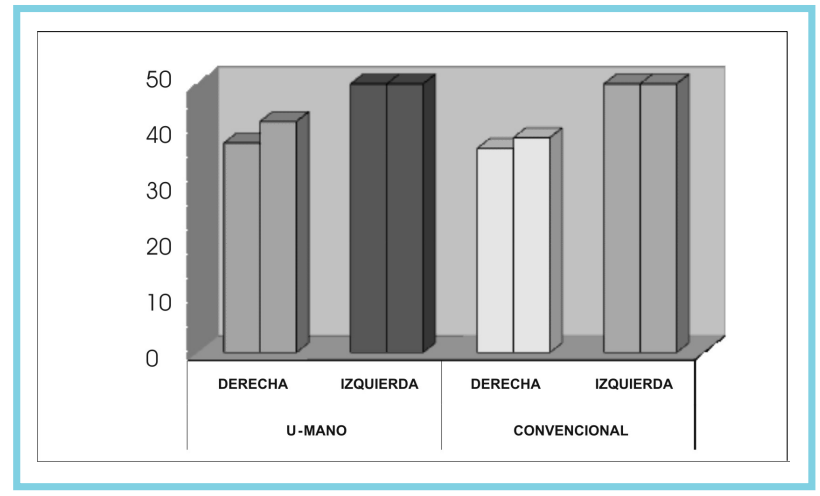

Gráfica 5. Funcionalidad de pinzas 
El dolor fue medido con la escala visual análoga del dolor; la valoración intermedia arrojó iguales resultados a la valoración final en todos los pacientes de ambos grupos. Aparecen los resultados de la primera valoración en la primera columna de cada mano, y en la segunda columna de cada mano aparece la información de la valoración final.

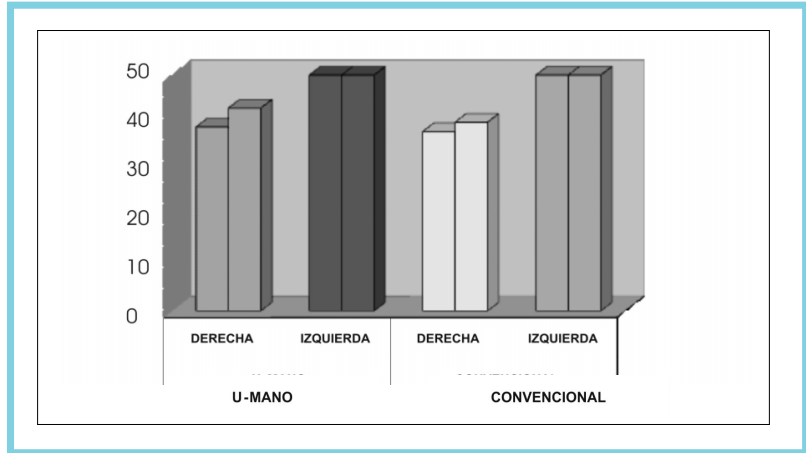

Gráfica 6. Dolor según la escala análoga visual

Todos los pacientes refirieron gran disminución del dolor en ambas manos. En especial se notó en manos izquierdas cuando hubo abolición del dolor. La disminución fue la misma para ambos grupos.

En ninguno de los pacientes de los dos grupos se encontraron alteraciones en la sensibilidad superficial mediante el test de monofilamentos en la valoración inicial.

Las parestesias y disestesias contabilizadas en las horas de la noche, cuantificadas en número de interrupciones del sueño por causa de los mismos, fue el factor que más sufrió modificaciones y que más motivó a los usuarios de ambos grupos a continuar con el tratamiento ocupacional. Los resultados se muestran en la gráfica 7. Los números de las columnas corresponden a la cantidad de veces que en promedio cada paciente se despertó durante la noche en la valoración inicial y en la valoración final. No se muestran los resultados de la valoración intermedia por ser exactos a la inicial.

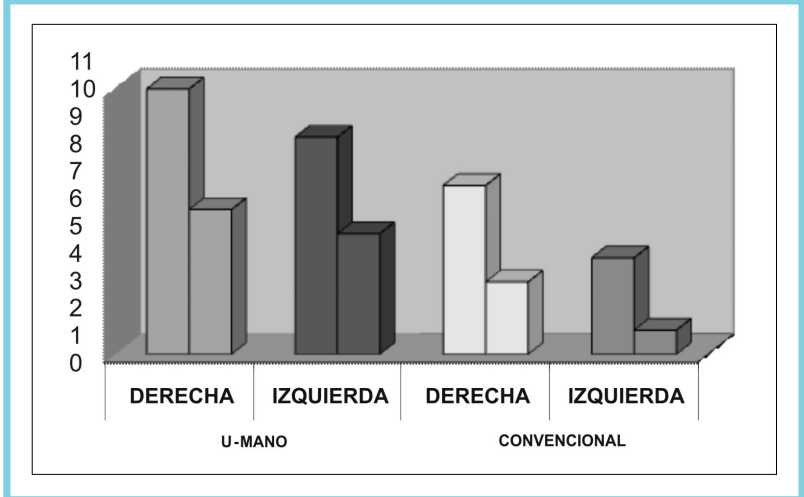

Gráfica 7. Parestesias nocturnas en los grupos.

El promedio de independencia en la totalidad de la prueba aumentó un 30,3\% para los usuarios de U-Mano, Mientras que para los usuarios de la férula convencional aumentó en un 6,66\%.

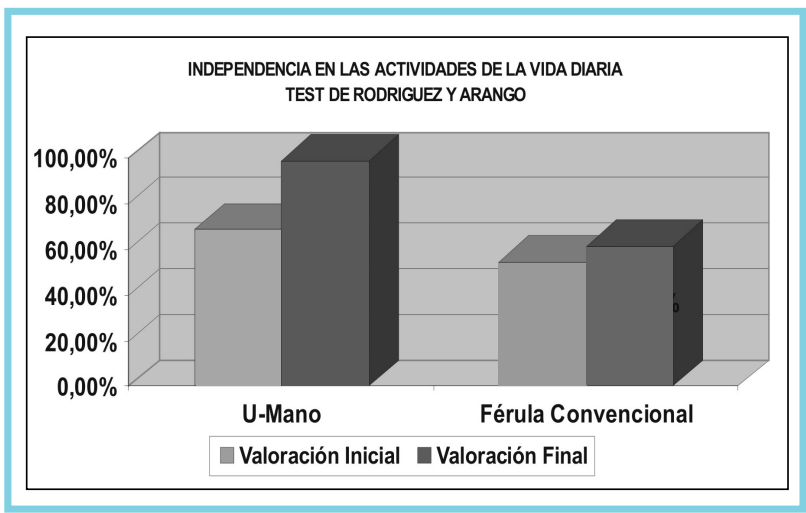

Gráfica 8. Independencia en las actividades de la vida diaria

\section{Discusión}

La ferula U-Mano fue más efectiva porque redujo de forma notoria los sintomas de STC. Adicionalmente es menos costosa y reportan los pacientes mayor comodidad con esta Férula.

Este trabajo fue desarrollado en su gran mayoría con usuarios del Hospital Militar Central, por lo cual se puede explicar la gran cantidad de usuarios auxiliares de enfermería o enfermeros. La medición de la independencia en las actividades de la vida diaria realizada con el test de Rodríguez Arango, no se en- 
cuentran diferencias en cuanto a las mediciones debido a que el protocolo de la férula convencional implica su utilización durante el día, haciendo que sea más difícil, y menos independiente la realización de las actividades de la vida diaria. Por otra parte debido a que los usuarios estaban diagnosticados todos con STC leve o moderado, aún no presentan atrofia marcada de sus eminencias tenares, ni alteraciones en la movilidad del pulgar o la sensibilidad superficial en la zona de inervación del nervio mediano.

\section{Conclusiones}

- La férula U-Mano es 6,5 veces menos costosa que la convencional; es también más cómoda aunque su durabilidad es menor que la convencional.

- La evaluación intermedia arrojó resultados exactamente iguales a la valoración inicial en todos los casos.

- El trofismo muscular no sufre modificación con ninguna de las dos férulas analizadas.

- El pulgar se encontró en todos los casos con los grados de movilidad completos tanto en la valoración inicial y como en la final.

- La funcionalidad de los agarres livianos, específicamente de la pinza trípode y lateral aumentó ligeramente en ambos grupos tras la intervención ocupacional.

- La sensibilidad protectiva estaba conservada en el 99\% de los casos desde el inicio. Tras la intervención ocupacional los pacientes del grupo U-Mano que mostraba hiperestesia y anestesia retornó a la sensibilidad normal.

- El dolor disminuyó 0.2 puntos en la escala verbal análoga del dolor en los pacientes del grupo de la férula U-Mano.

- Debido a que la férula U-Mano y la férula convencional ofrecieron igual beneficio, y la féru- la U-Mano es más económica, se recomienda implementar su uso.

Recibido: Febrero de 2007

Aceptado: Septiembre de 2007

\section{Referencias}

1. American Society for Surgery of the Hand. Hand: The examination and diagnosis of American Society for Surgery of the Hand. 1997.

2. Beck, L. Test de depresión. Disponible en la página Web: <http//www.depresion. psicomag.com/test.beck.php-62k.>

3. Bunnell-Boyes. Cirugía de la mano. Editorial Intermédica. 1967.

4. Cailliet, R. Síndromes dolorosos de la mano. Editorial El Manual Moderno. 1985

5. Cromwen, F. Hand rehabilitation in occupational therapy. 2000.

6. Fees, P. Hand splinting principles and methods. 1990.

7. Flynn, J.E. Hand surgery. Editorial El Ateneo. Buenos Aires. 2001

8. González, P. El Misterio de Protección Social STC. Revista El Cafelito. Diciembre de 2005.

9. Hamilton. S. Disponible en la página Web: $<$ http//www.depresion.psicomag.com/esc. hamilton.php>

10. Hernández-Sampieri, H. Metodología de la investigación. McGraw-Hill. Tercera edición. México. 2003.

11. Herrera, A., Fernández, L. et al. Monografías médico-quirúrgicas del aparato locomotor. Formación Continuada de la SECOT y de LASER. 1948-1998.

12. Hunter, A, Mackin, C. Callahan, T. Rehabilitation of the hand: surgery and therapy. Cuarta edición. 2002.

13. Iladiba, M.D. Actualización en cirugía de mano. Hand Clinics. 2002; 16 (9): 44-46. 
14. Iladiba, STC, revista. 2002; 16 (9): 44 p.

15. Iladiba, STC y computadores. 2003; 17 (6): $19 \mathrm{p}$.

16. Jacobson, J. Vitamin B6 Therapy for Carpal Tunnel Syndrome. Hand Clinics. Mayo, 1996; 12 (2): 253-257.

17. Kerwin, M.D. The pathophysiology of carpal tunnel syndrome. Hand Clinics. Mayo, 1996; 12 (2): 243-249.

18. Kasdan-Morton, L. Occupational hand upper extremity injuries. 1991.

19. Manente, M.D. Ann innovative hand brace for carpal tunnel syndrome: a randomized controlled trial. Muscle and Nerve Journal. Agosto, 2001; 1020- 1025.

20. Mcnaugthon, M.D. Overuse syndrome/ repetitive strain injury: the occupational therapist's role. British Journal of Occupational Therapy. Febrero, 1997.

21. Merler, M., Dantel, G. \& Loda, G. Mano traumática. Cirugía secundaria y muñeca traumática. Editorial Masson S.A, Barcelona, España. 1996.

22. Moran, C. Fisioterapia de la mano. Editorial Jims. Barcelona, España. 1990

23. Polit, H. Investigación científica en ciencias de la salud. McGraw-Hill. Sexta edición, México. 2000.

24. Rancel, STC: Diagnóstico y tratamiento. Revista ARP Colpatria Año 8 / Edición 32 / Diciembre de 2005.
25. Rasch, R \& Burke, T. Kinesiología y anatomía aplicada. 1996.

26. Rodríguez, S. STC. Guías clínicas. Marzo 9, 2000 .

27. Sailer, O.T. The role of splinting and rehabilitation in the treatment of carpal and cubital tunnel syndromes. Hand Clinics. Mayo, 1996; 12 (2. 223) 240 p.

28. Santaella, R y Herrera, L. Comparación de dos férulas de distinto material en cuanto a efectividad en amas de casa con STC. Revista De La Ocupación Humana, 1998; 7 (4): 13-37.

29. Szabo, M.D. Acute carpal tunnel syndrome. Hand Clinics. Ag. 1998; 14 (3): 419-427.

30. Szabo, M.D. Guía de manejo para el STC. Tribuna Médica. Mayo, 1998; 91, 265-268.

31. Viladot Perice, R. \& Cohi Riambau, O. Ortesis y prótesis del aparato locomotor 3 . Extremidad superior. Editorial Masson S.A., Barcelona 1992.

32. Von Schroeder, M.D. Carpal tunnel syndrome. Hand Clinics. Nov. 1996; 12 (4).

33. Wolfort, F. Lesiones agudas de la mano. 1993.

34. Zum, R. Escala autoaplicada de depresión de zung. Disponible en la página Web: <http// www.depresion.psicomag. com/esc.zung.php> 
